\title{
Glucagon-like peptide-1 analogues and risk of breast cancer in women with type 2 diabetes: population based cohort study using the UK Clinical Practice Research Datalink
}

\author{
Blánaid M Hicks, ${ }^{1,2}$ Hui Yin, ${ }^{1}$ Oriana H YYu, ${ }^{1,3}$ Michael N Pollak, ,,5 Robert W Platt, 1,2,6,7 \\ Laurent Azoulay ${ }^{1,2,4}$
}

${ }^{1}$ Centre for Clinical

Epidemiology, Lady Davis

Institute, Jewish General

Hospital, 3755 Côte Sainte-

Catherine, H-425.1, Montréal

H3T 1E2, Canada

${ }^{2}$ Department of Epidemiology,

Biostatistics and Occupational

Health, McGill University,

Montréal

${ }^{3}$ Division of Endocrinology,

Jewish General Hospital

${ }^{4}$ Gerald Bronfman Department of Oncology, McGill University

${ }^{5}$ Segal Cancer Center, Lady

Davis Institute for Medical

Research, Jewish General

Hospital

${ }^{6}$ Department of Pediatrics, McGill University

${ }^{7}$ Research Institute of the McGill University Health Centre,

Montreal

Correspondence to: L Azoulay laurent.azoulay@mcgill.ca

Additional material is published online only. To view please visit the journal online.

Cite this as: $B M$ J 2016;355:i5340 http://dx.doi.org/10.1136/bmj. i5340

Accepted: 23 September 2016

\section{ABSTRACT}

OBJECTIVE

To determine whether the use of glucagon-like peptide-1 (GLP-1) analogues, compared with the use of dipeptidylpeptidase-4 (DPP-4) inhibitors, is associated with an increased risk of incident breast cancer in patients with type 2 diabetes.

\section{DESIGN}

Population based cohort study.

SETTING

Clinical Practice Research Datalink, UK.

PARTICIPANTS

44984 women aged at least 40 years, who were newly treated with glucose lowering drugs between 1 January 2007 and 31 March 2015, with follow-up until 31 March 2016.

\section{MAIN OUTCOMES AND MEASURES}

Time varying use of GLP-1 analogues compared with use of DPP-4 inhibitors, with exposures lagged by one year for latency purposes. Time dependent Cox proportional hazards models were used to estimate adjusted hazard ratios with $95 \%$ confidence intervals of incident breast cancer associated with use of GLP-1 analogues overall, by cumulative duration of use, and time since initiation.

\section{RESULTS}

The cohort was followed for a mean of 3.5 years (standard deviation 2.2), with 549 incident events of breast cancer recorded (crude incidence 3.5 (95\% confidence interval 3.3 to 3.8) per 1000 person years). Overall, compared with use of DPP-4 inhibitors, use of

\section{WHAT IS ALREADY KNOWN ON THIS TOPIC}

Early randomised controlled trials of liraglutide, a glucagon-like peptide-1 (GLP-1) analogue, have reported imbalances in breast cancer events compared with placebo

Despite calls from regulatory agencies for further investigation, no observational studies have been conducted so far to analyse this safety concern

\section{WHAT THIS STUDY ADDS}

Use of GLP-1 analogues was not associated with an overall increased risk of breast cancer

Associations were observed with of GLP-1 analogue use, at two to three years and three to four years after initiation of treatment; however, these associations returned closer to the null with longer durations of treatment

Although it is not possible to rule out a tumour promoter effect, the observed duration-response associations are likely the result of a transient increase in detection of breast cancers in GLP-1 analogue users
GLP-1 analogues was not associated with an increased risk of breast cancer (incidence 4.4 V 3.4 per 1000 person years; hazard ratio 1.40 (95\% confidence interval 0.91 to 2.16$)$ ). Hazard ratios gradually increased with longer durations of use, with a peak between two to three years of GLP-1 use (2.66 (95\% confidence interval 1.32 to 5.38 )), and returned closer to the null after more than three years of use $(0.98$ (0.24 to 4.03)). A similar pattern was observed with time since initiation of GLP-1 analogues.

\section{CONCLUSIONS}

In this population based cohort study, use of GLP-1 analogues was not associated with an overall increased risk of breast cancer. Although it is not possible to rule out a tumour promoter effect, the observed duration-response associations are likely the result of a transient increase in detection of breast cancers in GLP-1 analogue users.

\section{Introduction}

Glucagon-like peptide-1 (GLP-1) analogues and dipeptidylpeptidase-4 (DPP-4) inhibitors are incretin based drugs used as second or third line treatments for type 2 diabetes. Although these drugs have been associated with favourable effects on hypoglycaemia and body weight, ${ }^{1}$ there have been concerns that their use could increase the risk of certain neoplasms, such as pancreatic cancer. ${ }^{23}$ There are also emerging concerns by regulatory agencies-including the US Food and Drug Administration and the European Medicines Agencythat GLP-1 analogues, in particular, might increase the risk of breast cancer. ${ }^{45}$

In randomised controlled trials of weight management, there were imbalances in the number of breast cancer events among patients randomised to the GLP-1 analogue liraglutide versus placebo (15 v 3 events; incidence $4.36 v 1.80$ per 1000 person years). ${ }^{56} \mathrm{~A}$ similar pattern was also observed in trials of patients with type 2 diabetes ( $9 \vee 1 ; 2.97 v 1.17$ per 1000 person years). ${ }^{5}$ However, these findings are at odds with those of the recently published LEADER trial (liraglutide effect and action in diabetes: evaluation of cardiovascular outcome results). ${ }^{7}$ In the LEADER trial, which randomised 9340 patients followed for a median of 3.8 years, researchers found no imbalances in breast cancer events among those randomised to liraglutide versus placebo $(21 / 1657(1.3 \%) v 20 / 1680(1.2 \%)){ }^{7}$

One hypothesis for the observed imbalance in the aforementioned randomised controlled trials relates to weight loss induced by GLP-1 analogues, possibly leading to a better detection of breast masses and 
increased uptake and accuracy of mammography. ${ }^{56}$ Indeed, the discrepancy between earlier trials and the recent LEADER trial might be due to a transient increase in detection of breast cancers relatively soon after treatment initiation. However, a competing hypothesis relates to a possible biological effect. Preclinical studies have shown that GLP-1 receptors are located on normal breast tissue ${ }^{8}$ and suggest that GLP-1 analogues could promote tumour growth via fibroblast growth factor 7 (FGF7). ${ }^{910}$

To our knowledge, no observational studies have been conducted to assess this important safety concern. Thus, this population based study aimed to assess whether the use of GLP-1 analogues to treat type 2 diabetes is associated with an increased risk of breast cancer.

\section{Methods}

\section{Data source}

This study was conducted by use of the UK Clinical Practice Research Datalink (CPRD). The CPRD includes data from about 700 general practices comprising over 14 million patients; this database has been shown to be representative of the UK population. ${ }^{11}$ The CPRD records demographic information, anthropometric data, lifestyle information, medical diagnoses and procedures, and prescription data that have been shown to be valid and of high quality. ${ }^{1213}$ Furthermore, breast cancer diagnoses recorded in the CPRD have been shown to be highly concordant $(>90 \%)$ with those recorded in the UK National Cancer Data Repository. ${ }^{14}$

The study protocol was approved by the independent scientific advisory committee of the CPRD (protocol number 16_096R) and by the research ethics board of the Jewish General Hospital, Montreal, Canada.

\section{Study population}

We identified all female patients, at least 40 years of age, who were newly prescribed a non-insulin glucose lowering drug (including metformin, sulfonylureas, prandial glucose regulators, thiazolidinediones, acarbose, DPP-4 inhibitors, GLP-1 analogues, and sodium-glucose cotransporter-2 inhibitors) between 1 January 1988 and 31 March 2015. Patients were required to have at least one year of medical history in the CPRD before their first prescription. We excluded those patients prescribed insulin at any time before their first prescription for a non-insulin glucose lowering drug, because this earlier prescription likely represented those individuals with advanced disease. Finally, we excluded female patients previously diagnosed with gestational diabetes or polycystic ovarian syndrome, because these are other indications for metformin.

Using the base cohort, we identified all patients who initiated a new glucose lowering drug class on or after 1 January 2007 (the year that the first incretin based drugs (sitagliptin and exenatide) entered the UK market) until 31 March 2015. These patients included those with a first ever diabetes drug prescription as well as those who added on or switched to a glucose lowering drug class not previously used in their treatment history. Cohort entry was defined by the date of this first prescription. Patients with a previous diagnosis of breast cancer (malignant and in situ) at any time before cohort entry were excluded. We also excluded patients with less than one year follow-up after cohort entry for latency purposes and to minimise detection bias due to increased contact with healthcare professionals at drug initiation. Consequently, patients diagnosed with breast cancer in the first year after cohort entry were not included in the cohort.

All patients meeting the study inclusion criteria were followed starting one year after cohort entry and until a first ever diagnosis of breast cancer (malignant and in situ, identified on the basis of Read codes; available on request), death from any cause, end of registration with the general practice, or the end of the study period (March 31, 2016), whichever occurred first.

\section{Exposure assessment}

We used a time dependent exposure definition. The exposure groups were defined hierarchically, with each person day classified into one of three mutually exclusive categories: GLP-1 analogues (exenatide, liraglutide and lixisenatide, alone or in combination with other glucose lowering drugs), DPP-4 inhibitors (sitagliptin, vildagliptin, and saxagliptin, alone or in combination with other glucose lowering drugs), and other glucose lowering drugs. All exposures were lagged by one year to account for a minimum latency period and to minimise reverse causality. Thus, patients initiating a glucose lowering drug were considered unexposed until one year after the date of the first prescription and considered exposed thereafter. The reference category was use of DPP-4 inhibitors, because these compounds are also incretin based drugs but have not been associated with breast cancer incidence in previous large randomised controlled trials. ${ }^{15-18}$ Furthermore, the first GLP-1 analogue and DPP-4 inhibitor were introduced in the UK in the same year (2007), and both drug classes are recommended as second to third line treatments, ${ }^{19}$ thereby minimising potential confounding by indication.

Based on the above exposure definition, we also defined the use of GLP-1 analogues in terms of cumulative duration of use and time since initiation. Cumulative duration of use was defined, in a time dependent fashion, as the total number of years of use calculated by summing the durations associated with each prescription between cohort entry and the risk set date (that is, time of the event). Time since initiation was defined, in a time dependent fashion, as the time between the first GLP-1 analogue prescription and the risk set date.

\section{Potential confounders}

All models were adjusted for the following variables measured at cohort entry: year of cohort entry, age, body mass index $(<25,25-29, \geq 30)$, smoking status (current, former, never, unknown), alcohol related disorders (including alcoholism, alcoholic cirrhosis of the liver, alcoholic hepatitis, and hepatic flexure), haemoglobin A1c (most recent laboratory result before 
cohort entry), and duration of treated diabetes (defined as the time between first non-insulin prescription and cohort entry).

The models were also adjusted for the presence of microvascular complications of diabetes (neuropathy, renal disease, retinopathy, and peripheral arteriopathy; measured at any time before cohort entry), and the number of unique glucose lowering drugs received in the year before cohort entry, both as proxies for diabetes severity. Additionally, we adjusted for previous oophorectomy, previous cancer diagnosis (excluding non-melanoma skin cancer), and the total number of unique non-glucose lowering drugs prescribed in the year before cohort entry (as a general measure of comorbidity ${ }^{20}$ ). Finally, all models were adjusted for the use of statins, hormonal replacement therapy, and oral contraceptives, measured at any time before cohort entry.

\section{Statistical analyses}

We used descriptive statistics to summarise the characteristics of the cohort. Crude incidence of breast cancer and $95 \%$ confidence intervals based on the Poisson distribution were calculated for the entire cohort, and separately for GLP-1 analogues and DPP-4 inhibitors. For the primary analysis, we used a time dependent Cox proportional hazards model to estimate hazard ratios and $95 \%$ confidence intervals of breast cancer associated with the use of GLP-1 analogues compared with DPP-4 inhibitor use. All models were adjusted for the potential confounders listed above.

\section{Secondary analyses}

We conducted three secondary analyses. The first assessed the risk associated with the most commonly used individual GLP-1 analogues (exenatide and liraglutide). The second assessed whether there was a duration-response association between cumulative duration of use of GLP-1 analogue and breast cancer incidence according to four predefined categories ( $\leq 1$ year, 1.1-2 years, 2.1-3 years, and $>3$ years). Thirdly, we assessed the association by time since initiation of GLP-1 analogues according to four predefined categories (1-2 years, 2.1-3 years, 3.1-4 years, and $>4$ years). In addition, we modelled both duration of use and time since initiation as continuous variables, using a restricted cubic spline model with five knots to produce a smooth curve of the hazard ratio as a function of these time variables. $^{2122}$

\section{Sensitivity analyses}

We conducted eight sensitivity analyses to assess the robustness of our findings:

- Given uncertainties related to the length of the latency time window, we repeated the primary analysis by lengthening the exposure lag period to two years.

- Because GLP-1 analogues are contraindicated among patients with renal failure, we repeated the primary analysis by also excluding patients with a history of renal disorders at any time before cohort entry and censoring on new diagnoses during follow-up.

- Because both GLP-1 analogues and DPP-4 inhibitors are incretin based treatments (and thus share similar mechanisms of action), we repeated the primary analysis using an alternate comparator group consisting of sulfonylureas. A similar analysis was also performed comparing DPP-4 inhibitors with sulfonylureas.

- We repeated the primary analysis using multiple imputation for variables with missing values (supplementary methods 1). ${ }^{2324}$

- We repeated the primary analysis also adjusting for the use of other glucose lowering drugs (including metformin, glyburide, other sulfonylureas, thiazolidinediones, insulin glargine, other insulins, and other glucose lowering drugs) during follow-up. For this analysis, these glucose lowering drugs were entered as non-mutually exclusive, time varying variables (lagged by one year) in addition to the main exposure variable.

- As another way to control for confounding, we repeated the analysis by stratifying the model in groups based on disease risk score (supplementary methods 2). .526

- To address any time dependent residual confounding during follow-up, we repeated the analysis using a marginal structural Cox proportional hazards model, which adjusts for time dependent confounding associated with time varying exposures (supplementary methods 3)..$^{27} 28$

- We conducted an analysis accounting for competing risk due to death using the subdistribution model proposed by Fine and Gray. ${ }^{29}$

\section{Analyses investigating detection bias}

Detection bias is a non-random type of information bias in which one exposure group might have a greater or lower opportunity of being screened for the outcome than another exposure group. ${ }^{30}$ To investigate the potential effect of detection bias on the findings, we conducted four analyses. Firstly, we repeated the primary analysis by removing the lag period (that is, to capture the early events after treatment initiation). Secondly, we repeated the primary analysis but restricted the outcome definition to malignant cancers and censoring on in situ breast cancers. Thirdly, we used inverse probability of screening weighting ${ }^{31}$ to account for the possibility that GLP-1 analogue users might have characteristics that increase or decrease their probability of undergoing mammography screening during follow-up (supplementary methods 4). Finally, we stratified the cohort based on the presence of at least one mammography screening in the three years before cohort entry (as per UK guidelines)..$^{32}$ For this analysis, we restricted the cohort to patients with at least three years of medical history before cohort entry in the CPRD, and estimated hazard ratios separately for patients with and without a screening history. ${ }^{33}$ All analyses described above were conducted with SAS version 9.4 (SAS institute). 


\section{Patient involvement}

Our study was a secondary data analysis and did not include patients as study participants. No patients were involved in setting the research question or the outcome measures, nor were they involved in the design and implementation of the study. The results will be disseminated to the general public through the public relations department of the Lady Davis Institute of the Jewish General Hospital, Montreal, Canada.

\section{Results}

The cohort included 44984 patients (fig 1), followed for a mean of 3.5 years (standard deviation 2.2). Overall, there were 549 incident events of breast cancer during 155071 person years of follow-up, generating a crude incidence of 3.5 (95\% confidence interval 3.3 to 3.8 ) per 1000 person years. A total of 2473 (5.5\%) patients were prescribed GLP-1 analogues during the study period, which included 1071 (43.3\%) liraglutide users, 792 $(32.0 \%)$ exenatide users, 98 (4.0\%) lixisenatide users, and $512(20.7 \%)$ who used multiple GLP-1 analogues during the follow-up period.

Table 1 presents baseline characteristics for the cohort, and by use of GLP-1 analogues, DPP-4 inhibitors, and other glucose lowering drugs at cohort entry. Compared with DPP-4 inhibitor users, GLP-1 analogue users were younger, less likely to have had alcohol related disorders, and more likely to be past smokers. GLP-1 analogue users were also more likely to have a higher body mass index, have a higher haemoglobin A1c level, and have used other glucose lowering drugs. Finally, GLP-1 analogue users were more likely to have used hormone replacement therapy and oral contraceptives, but less likely to have certain diabetes related complications and previous cancers.

Table 2 shows the results of primary and secondary analyses. Compared with DPP-4 inhibitor use, use of GLP-1 analogues was not associated with an overall

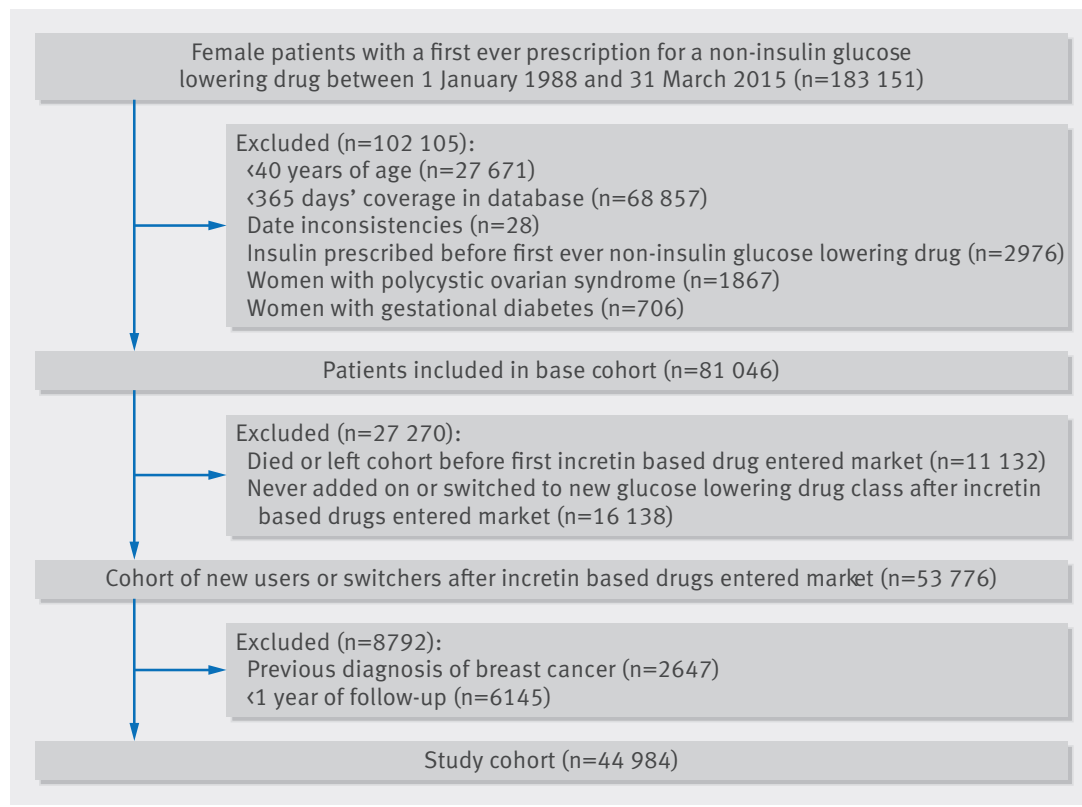

Fig 1 | Study flow diagram describing the construction of the base and study cohorts increased risk of breast cancer (4.4 v3.4 per 1000 person years; adjusted hazard ratio 1.40 (95\% confidence interval 0.91 to 2.16)). We found similar hazard ratios when analysing liraglutide and exenatide separately (hazard ratio 1.51 (95\% confidence interval 0.83 to 2.75$)$ and 1.33 ( 0.73 to 2.42 ), respectively). The model did not generate a stable hazard ratio for exposure to lixisenatide and combinations of the above, owing to few exposed events $(n=5)$.

In secondary analyses, 2.1 to 3 years of GLP-1 analogue use was associated with an increased risk of breast cancer (hazard ratio 2.66 (95\% confidence interval 1.32 to 5.38)), with the hazard ratio returning to the null after more than three years of use (0.98 (0.24 to 4.03)). We observed a similar pattern with time since initiation, where the hazard ratio was highest at 3.1-4 years after initiation (2.62 (1.37 to 4.99)), and retuned closer to the null after more than four years (1.14 (0.49 to 2.66)). These patterns were also observed in the restricted cubic spline analyses (supplementary figs 1 and 2).

\section{Sensitivity analyses}

Results of the sensitivity analyses are summarised in figure 2 and presented in supplementary tables 1-9. Overall, nearly all sensitivity analyses generated findings that were consistent with those of the primary analysis. An exception was the two year exposure lag analysis that led to a higher point estimate (hazard ratio 1.63 (95\% confidence interval 1.00 to 2.65$)$ ).

\section{Analyses investigating detection bias}

The analyses investigating possible detection bias are summarised in figure 3 and presented in supplementary tables 10-13. Removing the exposure lag window attenuated the hazard ratio (1.12 (95\% confidence interval 0.76 to 1.63$)$ ), whereas restricting the event definition to malignant breast cancers did not materially change the hazard ratio (1.47 (0.94 to 2.29)). Overall, the mammography screening rate was higher among GLP-1 analogue users than DPP-4 inhibitor users (18.6 (95\% confidence interval 17.7 to 19.5) $v 13.5$ (13.1 to 14.0 ) per 100 person years, respectively). Adjusting the models for the inverse probability of screening did not materially change the hazard ratio (1.36 (95\% confidence interval 0.75 to 2.48)). By contrast, we observed an increased risk of breast cancer among patients with no history of mammography screening (hazard ratio 1.94 (95\% confidence interval 1.08 to 3.50)) but no association among those with such a history (0.88 (0.45 to 1.75$)$ ).

\section{Discussion}

Principal findings

To our knowledge, this is the first observational study to investigate the association between the use of GLP-1 analogues and the risk of breast cancer in women with type 2 diabetes. In this study, the use of GLP-1 analogues was not associated with an overall increased risk of breast cancer. In secondary analyses, associations were observed with two to three years of use and with three to four years since initiation of treatment, but the asso- 


\begin{tabular}{|c|c|c|c|c|}
\hline \multirow[b]{2}{*}{ Characteristic } & \multirow[b]{2}{*}{$\begin{array}{l}\text { Entire } \\
\text { cohort }\end{array}$} & \multicolumn{3}{|l|}{ Subgroups } \\
\hline & & $\begin{array}{l}\text { Use of GLP-1 } \\
\text { analogues }\end{array}$ & $\begin{array}{l}\text { Use DPP-4 } \\
\text { inhibitors }\end{array}$ & $\begin{array}{l}\text { Use of other glucose } \\
\text { lowering drugs }\end{array}$ \\
\hline Total (No) & 44984 & $498(1.1)$ & $2422(5.4)$ & 42064 (93.5) \\
\hline Age (years; mean (SD)) & $64.8(12.0)$ & $59.8(8.4)$ & $67.8(10.4)$ & $64.7(12.0)$ \\
\hline Alcohol related disorders & $4817(10.7)$ & 64 (12.9) & 375 (15.5) & $4378(10.4)$ \\
\hline \multicolumn{5}{|l|}{ Smoking status } \\
\hline Current & 6405 (14.2) & $-^{\star}$ & $-^{\star}$ & $6094(14.5)$ \\
\hline Past & $13125(29.2)$ & $193(38.8)$ & $741(30.6)$ & $12191(29.0)$ \\
\hline Never & $25331(56.3)$ & $258(51.8)$ & $1415(58.4)$ & $23658(56.2)$ \\
\hline Unknown & $123(0.3)$ & $-^{\star}$ & $-^{\star}$ & $121(0.3)$ \\
\hline \multicolumn{5}{|l|}{ Body mass index } \\
\hline$<25$ & $4774(10.6)$ & $-^{\star}$ & $254(10.5)$ & $4516(10.7)$ \\
\hline $25-30$ & $11492(25.6)$ & $27(5.4)$ & $583(24.1)$ & $10882(25.9)$ \\
\hline$\geq 30.0$ & $27933(62.1)$ & $467(93.8)$ & $1578(65.2)$ & $25888(61.5)$ \\
\hline Unknown & $785(1.8)$ & $-^{\star}$ & $7(0.3)$ & $778(1.9)$ \\
\hline \multicolumn{5}{|l|}{ Haemoglobin A1c } \\
\hline$\leq 7.0 \%$ & 7669 (17.1) & 69 (13.9) & $304(12.6)$ & $7296(17.3)$ \\
\hline $7.1-8.0 \%$ & $13158(29.3)$ & $103(20.7)$ & $801(33.1)$ & 12254 (29.1) \\
\hline$>8.0 \%$ & $17900(39.8)$ & $321(64.5)$ & $1291(53.3)$ & $16288(38.7)$ \\
\hline Unknown & 6257 (13.9) & $5(1.0)$ & $26(1.1)$ & $6226(14.8)$ \\
\hline Duration of treated diabetes (years; mean (SD)) & $1.4(3.0)$ & $7.8(4.0)$ & $7.6(3.9)$ & $1.0(2.4)$ \\
\hline Neuropathy & $4792(10.7)$ & $134(26.9)$ & $625(25.8)$ & $4033(9.6)$ \\
\hline Renal disease & $8848(19.7)$ & $107(21.5)$ & $745(30.8)$ & $7996(19.0)$ \\
\hline Retinopathy & $9254(20.6)$ & $236(47.4)$ & $1243(51.3)$ & $7775(18.5)$ \\
\hline Peripheral arteriopathy & $1390(3.1)$ & $14(2.8)$ & $119(4.9)$ & $1257(3.0)$ \\
\hline Unique glucose lowering drugs (No; mean (SD)) & $0.3(0.7)$ & $2.1(0.9)$ & $1.6(0.8)$ & $0.2(0.6)$ \\
\hline \multicolumn{5}{|l|}{ Glucose lowering drugst } \\
\hline Metformin & $42217(93.9)$ & $475(95.4)$ & $2264(93.5)$ & 39478 (93.9) \\
\hline Sulfonylureas & $11451(25.5)$ & $364(73.1)$ & $1394(57.6)$ & $9693(23.0)$ \\
\hline Thiazolidinediones & $4442(9.9)$ & $253(50.8)$ & $912(37.7)$ & $3277(7.8)$ \\
\hline Insulins & $1411(3.1)$ & $156(31.3)$ & $108(4.5)$ & $1147(2.7)$ \\
\hline Other glucose lowering drugs & $515(1.1)$ & $44(8.8)$ & $113(4.7)$ & $358(0.9)$ \\
\hline Oophorectomy & $1681(3.7)$ & $19(3.8)$ & $107(4.4)$ & $1555(3.7)$ \\
\hline Cancer & $4346(9.7)$ & $38(7.6)$ & $274(11.3)$ & $4034(9.6)$ \\
\hline Unique non-diabetic drugs (No; mean (SD)) & $9.7(6.5)$ & $13.6(7.1)$ & $11.8(6.6)$ & $9.5(6.4)$ \\
\hline Statins & $31884(70.9)$ & $441(88.6)$ & 2219 (91.6) & $29224(69.5)$ \\
\hline Hormonal replacement therapy & $13918(30.9)$ & $207(41.6)$ & $894(36.9)$ & $12817(30.5)$ \\
\hline Oral contraceptives & 6118 (13.6) & $78(15.7)$ & $220(9.1)$ & $5820(13.8)$ \\
\hline
\end{tabular}

\begin{tabular}{|c|c|c|c|c|c|}
\hline Exposure* & $\begin{array}{l}\text { No of breast } \\
\text { cancer events }\end{array}$ & $\begin{array}{l}\text { No of person } \\
\text { years }\end{array}$ & $\begin{array}{l}\text { Incidence }(95 \% \mathrm{Cl}) \\
\text { per } 1000 \text { person years }\end{array}$ & $\begin{array}{l}\text { Crude hazard } \\
\text { ratio }(95 \% \mathrm{Cl})\end{array}$ & $\begin{array}{l}\text { Adjusted hazard } \\
\text { ratio }(95 \% \mathrm{Cl}) \dagger\end{array}$ \\
\hline DPP-4 inhibitors & 68 & 19906 & $3.4(2.7$ to 4.3$)$ & 1.00 (reference) & 1.00 (reference) \\
\hline GLP-1 analogues & 31 & 6983 & 4.4 (3.0 to 6.3$)$ & 1.29 (0.85 to 1.98$)$ & 1.40 (0.91 to 2.16$)$ \\
\hline \multicolumn{6}{|c|}{ Type of GLP-1 analogue } \\
\hline Exenatide only & 13 & 3090 & $4.2(2.2$ to 7.2$)$ & 1.23 (0.68 to 2.23$)$ & $1.33(0.73$ to 2.42$)$ \\
\hline Liraglutide only & 13 & 2724 & $4.8(2.5$ to 8.2$)$ & 1.39 (0.77 to 2.52$)$ & 1.51 (0.83 to 2.75$)$ \\
\hline Other & 5 & 1169 & $4.3(1.4$ to 10.0$)$ & $1.24(0.50$ to 3.07$)$ & 1.34 (0.54 to 3.34$)$ \\
\hline \multicolumn{6}{|c|}{ Cumulative duration of GLP-1 analogue use (years) } \\
\hline$\leq 1$ & 11 & 2921 & $3.8(1.9$ to 6.7$)$ & $1.10(0.58$ to 2.09$)$ & 1.21 (0.64 to 2.30$)$ \\
\hline $1.1-2$ & $-\S$ & $-\S$ & $3.8(1.7$ to 7.2$)$ & $1.10(0.55$ to 2.20$)$ & 1.18 (0.59 to 2.38$)$ \\
\hline $2.1-3$ & 9 & 1051 & $8.6(3.9$ to 16.3$)$ & 2.49 (1.24 to 5.01$)$ & $2.66(1.32$ to 5.38$)$ \\
\hline$>3$ & $-\S$ & $-\S$ & $3.2(0.4$ to 11.5$)$ & $0.92(0.22$ to 3.75$)$ & $0.98(0.24$ to 4.03$)$ \\
\hline \multicolumn{6}{|c|}{ Time since first GLP-1 analogue use (years) } \\
\hline $1-2$ & 5 & 2237 & $2.2(0.7$ to 5.2$)$ & $0.66(0.27$ to 1.64$)$ & $0.72(0.29$ to 1.78$)$ \\
\hline $2.1-3$ & 9 & 1775 & $5.1(2.3$ to 9.6$)$ & $1.47(0.73$ to 2.94$)$ & 1.59 (0.79 to 3.21$)$ \\
\hline $3.1-4$ & 11 & 1324 & $8.3(4.1$ to 14.9$)$ & $2.42(1.28$ to 4.58$)$ & 2.62 (1.37 to 4.99$)$ \\
\hline$>4$ & 6 & 1647 & $3.6(1.3$ to 7.9$)$ & 1.05 (0.45 to 2.45$)$ & 1.14 (0.49 to 2.66$)$ \\
\hline \multicolumn{6}{|c|}{$\begin{array}{l}\text { DPP-4=dipeptidyl peptidase-4; GLP-1=glucagon-like peptide-1. } \\
\text { *Use of other diabetes drugs (generating } 450 \text { breast events) were considered in the models for proper estimation of effects, but not presented in the } \\
\text { table. } \\
\text { tAdjusted for age, year of cohort entry, body mass index, smoking status, alcohol related disorders, haemoglobin A1c, duration of treated diabetes } \\
\text { (measured at cohort entry); use of statins, hormonal replacement therapy, and oral contraceptives (measured at any time before cohort entry); presence } \\
\text { of microvascular complications of diabetes (neuropathy, renal disease, retinopathy, and peripheral arteriopathy; measured at any time before study } \\
\text { cohort entry); the number of unique glucose lowering drugs received in the year before cohort entry; previous cancer diagnosis (excluding non- } \\
\text { melanoma skin cancer), previous oophorectomy, and the total number of unique non-diabetic drugs prescribed in the year before cohort entry. } \\
\text { §Cells with less than five observations are not displayed, as per the confidentiality policies of the Clinical Practice Research Datalink. }\end{array}$} \\
\hline
\end{tabular}




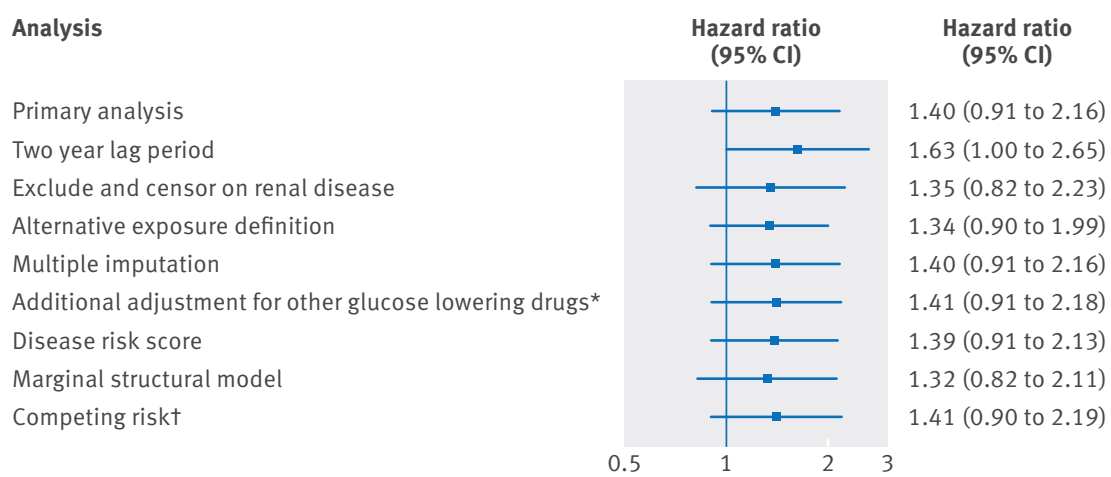

Fig 2 | Forest plot summarising results of the primary and sensitivity analyses assessing the association between GLP-1 analogue use and breast cancer incidence. *Other glucose lowering drugs entered as non-mutually time varying covariates in addition to the original three level exposure variable. †Estimate is a subdistribution hazard ratio

Analysis
No lag period
Restricted event definition*
Inverse probability of screening weighting
History of previous screening
No history of previous screening

0.2

Fig 3 | Forest plot summarising results of the analyses investigating possible detection bias of breast cancer with GLP-1 analogue use. *Event definition restricted to women with malignant breast cancers

ciations returned closer to the null with longer durations of use and times since initiation. Although these atypical duration patterns are compatible with a possible tumour promoter effect, they are likely a result of increased detection.

\section{Comparison with previous studies}

Supporting the hypothesis of increased detection are the results of the recent LEADER trial in which no imbalance in breast cancer events was observed between patients randomised to liraglutide versus placebo. ${ }^{7}$ Although not designed to investigate this safety endpoint, the LEADER trial had a longer follow-up period than previous randomised controlled trials $(<1$ year), suggesting that initial imbalances in breast cancer might have been the result of a transient detection bias. This result is consistent with those of our study, where associations were observed with two to three year durations of use and three to four years since initiation of GLP-1 analogue use, but the estimates returned closer to the null with longer durations. Furthermore, in analyses investigating the possibility of detection bias, the association was limited to those women with no history of mammography screening-a finding that is compatible with a possible enhanced detection of prevalent disease.

The mechanism for this possible detection bias is not well understood. Although it has been proposed that weight loss induced by GLP-1 analogues could lead to enhanced detection of breast lumps and increased uptake and accuracy of mammography, evidence supporting this hypothesis is lacking, ${ }^{3435}$ and thus additional studies are needed to investigate this hypothesis. Given the observational nature of this study, body weight was not recorded in a systematic fashion, and therefore it was not possible to evaluate the effect of rapid weight loss on the detection of breast cancer. However, it would be of interest to perform such analyses using data from recent large randomised controlled trials of GLP-1 analogues (eg, SCALE $^{6}$ and LEADER ${ }^{7}$ trials), where body weight was measured in a systematic fashion before randomisation and during follow-up.

Although detection bias is a likely explanation for the observed results, a tumour promoter effect of GLP-1 analogues cannot be ruled out. GLP-1 analogues can promote tumour growth via increased expression of fibroblast growth factor 7 (FGF7). ${ }^{9}$ GLP-1 receptors have been located on human breast tissue, ${ }^{8}$ and FGF7 is known to be an important paracrine growth factor within the breast. ${ }^{36}$ Moreover, its receptor FGFR2 is overexpressed in human breast cancer tissue ${ }^{10}$ and is known to be a breast cancer susceptibility gene; its variants associated with breast cancer risk. ${ }^{37}$ Thus, the interaction between GLP-1 analogues and the FGF7/ FGFR2 axis could stimulate proliferation of a subset of early breast cancers. Additional studies are needed to determine the exact mechanisms through which GLP-1 analogues might increase the risk of breast cancer.

\section{Strengths and limitations}

This study has several strengths. Firstly, the use of a new user design minimised biases related to the inclusion of prevalent users. ${ }^{38}$ Secondly, we used a time dependent exposure definition, and the exposures were lagged for latency purposes. Additionally, the models were 
adjusted for several potential confounders, and a marginal structural model analysis was conducted to adjust for potential time dependent confounders, yielding similar results. Finally, with the availability of mammography screening information, we were able to conduct various analyses to investigate possible detection bias.

This study also has some limitations. Prescriptions in the CPRD represent those written by general practitioners, and thus misclassification of exposure is possible. However, since all patients entering the cohort were those newly treated with glucose lowering drugs, misclassification is likely minimal and non-differential between exposure groups. Secondly, given the observational nature of the study, residual confounding from unmeasured or unknown variables (such as family history of breast cancer) is possible. Misclassification of the outcome is also possible, although breast cancer has been shown to be well recorded in the CPRD when compared with the UK National Cancer Data Repository. ${ }^{14}$ GLP-1 analogues are relatively new drugs, limiting the potential duration of follow-up (maximum of about 9.5 years), which could be considered too short to investigate cancer risk. Indeed, exposure to GLP-1 analogues yielded a relatively small number of breast cancer events $(n=31)$, thus potentially limiting statistical power. However, the rationale for conducting the present study was based on the imbalances observed in randomised controlled trials, which had relatively short durations (20-56 weeks). ${ }^{5}$

\section{Conclusions}

In this population based study, the use of GLP-1 analogues was not associated with an overall increased risk of breast cancer risk, providing some reassurance to patients and clinicians. While the observed duration-response associations might be compatible with a tumour promoter effect, they are likely a result of a transient increase in detection of breast cancers in GLP-1 analogue users.

Contributors: All authors conceived and designed the study, analysed and interpreted the data, and critically revised the manuscript for important intellectual content. LA acquired the data. BMH, HY, RWP, and LA did the analyses. BMH wrote the manuscript and all authors participated in the interpretation of the results and critical revision of the manuscript. LA is the guarantor.

Funding: This study was funded by a foundation grant from the Canadian Institutes of Health Research. The sponsor had no influence on design and conduct of the study; collection, management, analysis, and interpretation of the data; and preparation, review, or approval of the manuscript.

Competing interests: All authors have completed the ICMJE uniform disclosure form at www.icmje.org/coi_disclosure.pdf and declare: support from the Canadian Institutes of Health Research for the submitted work; RWP holds the Albert Boehringer I chair and is the recipient of a Chercheur-National career award from the Fonds de Recherche du Quebec-Santé (FRQS; Quebec Foundation for Health Research); RWP also reports personal fees from Pfizer, Novartis, Abbvie, and Amgen, outside the submitted work; LA is the recipient of a Chercheur-Boursier career award from the FRQS; all other authors have no financial relationships with any organisations that might have an interest in the submitted work in the previous three years, and no other relationships or activities that could appear to have influenced the submitted work.

Ethical approval: The study protocol was approved by the independent scientific advisory committee of the Clinical Practice Research Datalink (protocol number 16_096R) and by the research ethics board of Jewish General Hospital, Montreal, Quebec, Canada. Data sharing: No additional data available.
The lead author (the manuscript's guarantor) affirms that the manuscript is an honest, accurate, and transparent account of the study being reported; that no important aspects of the study have been omitted; and that any discrepancies from the study as planned (and, if relevant, registered) have been explained.

This is an Open Access article distributed in accordance with the Creative Commons Attribution Non Commercial (CC BY-NC 3.0) license, which permits others to distribute, remix, adapt, build upon this work non-commercially, and license their derivative works on different terms, provided the original work is properly cited and the use is non-commercial. See: http://creativecommons.org/licenses/ by-nc/3.0/.

Holst JJ. The physiology of glucagon-like peptide 1. Physiol Rev 2007;87:1409-39. doi:10.1152/physrev.00034.2006.

2 Łabuzek K, Kozłowski M, Szkudłapski D, Sikorska P, Kozłowska M, Okopień B. Incretin-based therapies in the treatment of type 2 diabetes-more than meets the eye?Eur J Intern Med 2013;24:207-12. doi:10.1016/j.ejim.2013.01.009.

3 Gale EAM. GLP-1-based therapies and the exocrine pancreas: more light, or just more heat?Diabetes 2012;61:986-8. doi:10.2337/ db11-1838.

4 European Medical Agency Committee for Medicinal Products for Human Use. Assessment report Saxenda International nonproprietary name: liraglutide. 2015. www.ema.europa.eu/docs/ en_GB/document_library/EPAR_-_Public_assessment_report/ human/003780/WC500185788.pdf

5 FDA. Analysis of cancer incidence observed in clinical trials of liraglutide. 2016. www.fda.gov/downloads/advisorycommittees/ committeesmeetingmaterials/drugs/endocrinologicand metabolicdrugsadvisorycommittee/ucm416154.pdf

6 Pi-Sunyer X, Astrup A, Fujioka K, et al. SCALE Obesity and Prediabetes NN8022-1839 Study Group. A randomized, controlled trial of 3.0 mg of liraglutide in weight management. N Engl J Med 2015;373:11-22. doi:10.1056/NEIMoa1411892.

7 Marso SP, Daniels GH, Brown-Frandsen K, et al. LEADER Steering Committee LEADER Trial Investigators. Liraglutide and Cardiovascular Outcomes in Type 2 Diabetes. N Engl / Med 2016;375:311-22. doi:10.1056/NEJMoa1603827.

8 Körner M, Stöckli M, Waser B, Reubi JC. GLP-1 receptor expression in human tumors and human normal tissues: potential for in vivo targeting. J Nucl Med 2007:48:736-43. doi:10.2967/ jnumed.106.038679.

9 Koehler JA, Baggio LL, Yusta B, et al. GLP-1R agonists promote normal and neoplastic intestinal growth through mechanisms requiring Fgf7. Cell Metab 2015;21:379-91. doi:10.1016/j.cmet.2015.02.005.

10 Tannheimer SL, Rehemtulla A, Ethier SP. Characterization of fibroblast growth factor receptor 2 overexpression in the human breast cancer cell line SUM-52PE. Breast Cancer Res 2000;2:311-20. doi:10.1186/ bcr73.

11 Herrett E, Gallagher AM, Bhaskaran K, et al. Data Resource Profile: Clinical Practice Research Datalink (CPRD). Int J Epidemiol 2015;44:827-36. doi:10.1093/ije/dyv098.

12 Jick SS, Kaye JA, Vasilakis-Scaramozza C, et al. Validity of the general practice research database. Pharmacotherapy 2003;23:686-9. doi:10.1592/phco.23.5.686.32205.

13 Lawrenson R, Williams T, Farmer R. Clinical information for research; the use of general practice databases. J Public Health Med 1999.21:299-304 doi:10.1093/pubmed/21.3.299.

14 Boggon R, van Staa TP, Chapman M, Gallagher AM, Hammad TA, Richards MA. Cancer recording and mortality in the General Practice Research Database and linked cancer registries. Pharmacoepidemiol Drug Saf 2013;22:168-75. doi:10.1002/pds.3374

15 Scirica BM, Bhatt DL, Braunwald E, et al. SAVOR-TIMI 53 Steering Committee and Investigators. Saxagliptin and cardiovascular outcomes in patients with type 2 diabetes mellitus. N Engl / Med 2013;369:1317-26. doi:10.1056/NEJMoa1307684.

16 Leiter LA, Teoh H, Mosenzon O, et al. SAVOR-TIMI 53 Steering Committee and Investigators. Frequency of cancer events with saxagliptin in the SAVOR-TIMI 53 trial. Diabetes Obes Metab 2016;18:186-90. doi:10.1111/dom.12582.

17 White WB, Cannon CP, Heller SR, et al. EXAMINE Investigators. Alogliptin after acute coronary syndrome in patients with type 2 diabetes. $N$ EnglJ Med 2013;369:1327-35. doi:10.1056/NEJMoa1305889.

18 Green JB, Bethel MA, Armstrong PW, et al. TECOS Study Group. Effect of Sitagliptin on Cardiovascular Outcomes in Type 2 Diabetes. N Engl Med 2015;373:232-42. doi:10.1056/NEJMoa1501352.

19 The National Institute of Heath and Care Excellence. Type 2 diabetes in adults: management -Recommendations Guidance and guidelines [Internet] 2015 [Cited 2016 April 19] Available from https://www.nice. org.uk/guidance/ng28/evidence

20 Schneeweiss S, Seeger JD, Maclure M, Wang PS, Avorn J, Glynn RJ. Performance of comorbidity scores to control for confounding in epidemiologic studies using claims data. Am J Epidemiol 2001;154:854-64. doi:10.1093/aje/154.9.854 
21 Greenland S. Dose-response and trend analysis in epidemiology: alternatives to categorical analysis. Epidemiology 1995;6:356-65. doi:10.1097/00001648-199507000-00005.

22 Heinzl H, Kaider A, Zlabinger G. Assessing interactions of binary time-dependent covariates with time in cox proportional hazards regression models using cubic spline functions. Stat Med 1996;15:2589-601. doi:10.1002/(SICI)1097-0258(19961215) 15:23<2589::AID-SIM373>3.0.CO;2-0.

23 Sschafer JL. Analysis of incomplete multivariate data. statistics in medicine.Chapman and Hall, 1997:1006-08.

24 Rubin DB, ed. Multiple Imputation for Nonresponse in Surveys.John Wiley \& Sons, Inc, 1987doi:10.1002/9780470316696.

25 Arbogast PG, Ray WA. Performance of disease risk scores, propensity scores, and traditional multivariable outcome regression in the presence of multiple confounders. Am J Epidemiol 2011;174:613-20. doi:10.1093/aje/kwr143.

26 Arbogast PG, Ray WA. Use of disease risk scores in pharmacoepidemiologic studies. Stat Methods Med Res 2009;18:6780. doi:10.1177/0962280208092347.

27 Hernán MA, Brumback B, Robins IM. Marginal structural models to estimate the causal effect of zidovudine on the survival of HIV-positive men. Epidemiology 2000;11:561-70. doi:10.1097/00001648-200009000-00012.

28 Robins JM, Hernán MA, Brumback B. Marginal structural models and causal inference in epidemiology. Epidemiology 2000;11:550-60. doi:10.1097/00001648-200009000-00011

29 Fine IP, Gray RJ. A proportional hazards model for the subdistribution of a competing risk. J Am Stat Assoc 1999;94:496-509doi:10.1080/01621459.1999.10474144.

30 Haut ER, Pronovost PJ. Surveillance bias in outcomes reporting. JAMA 2011:305:2462-3. doi:10.1001/jama.2011.822.
31 Cook NR, Rosner BA, Hankinson SE, Colditz GA. Mammographic screening and risk factors for breast cancer. Am J Epidemiol 2009:170:1422-32. doi:10.1093/aje/kwp304.

32 Public Health England. NHS breast screening programme [Internet] Available from: https://www.gov.uk/topic/population-screeningprogrammes/breast

33 Joffe MM, Colditz GA. Restriction as a method for reducing bias in the estimation of direct effects. Stat Med 1998;17:2233-49. doi:10.1002/(SICl)1097-0258(19981015)17:19<2233::AID $\mathrm{SIM} 922>3.0 \mathrm{CO} \cdot 2-0$

34 Njor SH, von Euler-Chelpin M, Tjønneland A, Vejborg I, Lynge E. Body weight and sensitivity of screening mammography. Eur J Cancer 2016;60:93-100. doi:10.1016/j.ejca.2016.02.028.

35 Elmore JG, Carney PA, Abraham LA, et al. The association between obesity and screening mammography accuracy. Arch Intern Med 2004;164:1140-7. doi:10.1001/archinte.164.10.1140.

36 Palmieri C, Roberts-Clark D, Assadi-Sabet A, et al. Fibroblast growth factor 7 , secreted by breast fibroblasts, is an interleukin-1 beta induced paracrine growth factor for human breast cells. J Endocrinol 2003;177:65-81. doi:10.1677/joe.0.1770065.

37 Cui F, Wu D, Wang W, He X, Wang M. Variants of FGFR2 and their associations with breast cancer risk: a HUGE systematic review and meta-analysis. Breast Cancer Res Treat 2016;155:313-35. doi:10.1007/s10549-015-3670-2.

38 Ray WA. Evaluating medication effects outside of clinical trials: new-user designs. Am J Epidemiol 2003;158:915-20. doi:10.1093/ aje/kwg231

Supplementary appendix: Additional material 